\title{
Pesquisa qualitativa em design: protocolos de pesquisa como suporte a criação e avaliação de produtos conceituais
}

\author{
Dioclecio Moreira Camelo ${ }^{1}$, Andréa Grano Marques², Amanda Roque ${ }^{1}$, Andreza Vieira ${ }^{1}$ \\ e Isabela Bellido ${ }^{1}$
}

dmcamelo@uem.br, andreagrano298@hotmail.com, roque.amandas@gmail.com, andrezavieira@hotmail.com, isabela.m.belido@gmail.com

${ }^{1}$ Laboratório Avançado sobre Estudos em Design e Inovação da Universidade Estadual de Maringá, CEP 87200-055, Cianorte - Paraná, Brasil.

${ }^{2}$ Docente do Programa de Pós-Graduação em Promoção da Saúde do Centro Universitário de Maringá - UNICESUMAR; Pesquisadora, Bolsista Produtividade em Pesquisa do Instituto Cesumar de Ciência, Tecnologia e Inovação (ICETI), CEP 87050-900, Maringá - Paraná, Brasil.

DOI: 10.17013/risti.23.78-89

\begin{abstract}
Resumo: Os designers, ao longo do processo de desenvolvimento de novos produtos, deparam-se com o fenômeno de fixação de ideias ao reproduzir características de produtos ou ao repetir soluções conceituais do projeto. O objetivo do presente trabalho foi apresentar dois protocolos de pesquisa propostos para identificar o grau de influência das informações ambientais sobre o processo criativo de designers, empregando-se métodos qualitativos. A primeira fase da pesquisa permitiu definir o protocolo de orientação aos pesquisadores para a realização de práticas simuladas de projeto e, posteriormente, a elaboração do protocolo de suporte de avaliação das ideias conceituais geradas por designers. Este protocolo foi elaborado em dois ciclos de desenvolvimentos, o primeiro ciclo estabeleceu instrumentos para avaliar os aspectos sobre o critério de novidade dos produtos enquanto o segundo ciclo acrescentou o critério de utilidade do produto. Foram realizados testes pilotos de aplicação dos protocolos, sendo reformulados validando a aplicabilidade dos instrumentos.
\end{abstract}

Palavras chave: Criatividade; Design de produtos; Fixação de idéias; Informação ambiental; Pesquisa qualitativa.

\section{Qualitative research in design: protocols to support the creation and evaluation of conceptual products}

\begin{abstract}
Throughout the process of developing new products, designers are faced with the idea-fixation phenomenon by reproducing product characteristics or by repeating conceptual project solutions. The objective of the present work was to present two research protocols proposed to identify the degree of influence of environmental information on the creative process of designers, using qualitative methods. The first phase of the research defined the protocol to guide researchers
\end{abstract}


on simulated practices of project and later on the evaluation of conceptual ideas generated by groups of designers. This protocol was elaborated in two development cycles, the first cycle established instruments to evaluate the criterion of novelty of the products while the second cycle added the criterion of utility of the product. Pilot tests were carried out to apply the protocols, being reformulated validating the applicability of the instruments.

Keywords: Creativity; Product Design; Design fixation; Environmental Information; Qualitative research.

\section{Introdução}

Ao longo do processo de desenvolvimento de novos produtos os designers consultam e compilam informações sobre diversos produtos, buscando soluções inovadoras para a concepção do novo produto que será levado ao mercado. O designer ao reproduzir características dos produtos consultados ou repetir soluções conceituais do projeto deparam-se com o fenômeno de fixação de ideias.

Estima-se que diversos fatores podem intervir no processo criativo e contribuir para a fixação de ideias, alguns desses fatores estão relacionados com a formação do profissional que está envolvido no desenvolvimento do produto e o nível de detalhamento das informações (soluções, processos e materiais) disponíveis para os designers, assim como as técnicas de criatividade adotadas para gerar novas ideias. As causas que explicam o fenômeno não estão restritas somente a estes fatores. Crilly (2015) amplia as possíveis causas que podem conduzir os designers a reproduzir o fenômeno de fixação de ideias, dentre elas estão a consulta a soluções anteriores, a fixação às ideias iniciais e às informações contidas no briefing.

Purcell e Gero (1996) apontaram que a formação dos projetistas envolvidos no desenvolvimento e os diferentes tipos de soluções consultadas podem interferir na criatividade das propostas ao longo do desenvolvimento do projeto. Collado-Ruiz e Ostad-Ahmad-Ghorabi (2010) identificaram que o uso de informação ambiental sobre os produtos e os processos de produção, tais como a análise do ciclo de vida do produto, as emissões contabilizadas na produção, assim como o uso e o descarte do produto influenciam no resultado criativo e nas características inovadoras, entretanto, no processo de avaliação das ideias os autores consideraram critérios subjetivos para analisar as propostas desenvolvidas por equipes de designers. LopezMesa (2011) identificou que diferentes técnicas utilizadas para a geração de ideias interferem no grau de criatividade empregado no desenvolvimento de produtos inovadores.

As causas que podem influenciar no processo criativo dos designers precisam ser investigadas com maior profundidade, utilizando-se métricas que permitam avaliar os produtos a fim de encontrar relação de causa e efeito entre as informações consultadas e o grau de criatividade alcançado nas propostas dos produtos, com isto, seria possível identificar as causas que possam levar designers a fixar ideias e reproduzir soluções. Portanto, é necessário estabelecer protocolos que possibilitem a simulação do desenvolvimento de produtos em ambiente controlado, como também a avaliação do grau de criatividade empregado na concepção de novos produtos. 
Nos estudos em design alguns pesquisadores utilizam métodos quantitativos para comprovar hipóteses sobre as causas que conduzem os designers a fixação de ideias, outros empregam métodos qualitativos para analisar as possíveis causas ou tentam identificar a relação entre o estímulo e a geração de ideias criativas. Na aplicação de métodos qualitativos dentro do universo dos designers, podemos citar o trabalho de Lopez-Mesa (2011) que utiliza da análise de protocolo aplicando uma codificação sistemática a partir de símbolos e fluxos de atividades para acompanhar a interação entre os designers de uma equipe. Esta análise de protocolo foi utilizada como base para descrever a influência das técnicas de geração de ideias sobre o processo criativo dos designers. Daros (2013) aplica ciclos de treinamento e análise de múltiplos instrumentos de coleta de dados de consumidores (vídeos, áudios de entrevistas e sondas culturais), considerando a participação de equipes multidisciplinares para analisar esse material e identificar hábitos de consumo.

Estudos de outras áreas também adotam diferentes formas para a análise das narrativas e das entrevistas gravadas em áudio ou vídeos. Vosgerau Pocrifka e Simonian (2016) propuseram uma associação de ferramentas para a coleta de dados, codificação e análise dos resultados, utilizando-se do software ATLAS.ti para a pré-análise e codificação dos conteúdos, a pré-análise sistematizou e operacionalizou as ideias, enquanto que a codificação consistiu em escolher as unidades de sentido presente nos discursos. Contreras et al. (2017) utilizou a combinação de métodos qualitativos e quantitativos para auxiliar no aprimoramento de materiais de ensino. Sob a ótica qualitativa, foi adotada a análise textual e o uso do Software WQA para identificar as partes que precisavam ser aprimoradas. García-Merino (2017) aplicou múltiplos métodos para auxiliar na análise do discurso e na interpretação corporal de diretores a partir de imagens registradas em vídeos corporativos para explicar as estratégias empresariais. Silva e Correa (2017) aplicaram a análise do discurso como ferramenta para refletir sobre as práticas de ensino à partir de vídeos gravados em salas de aulas. Os métodos qualitativos podem ser empregados em diversas áreas do conhecimento com o auxílio de ferramentas de software para préanálise, categorização e análise dos dados coletados (Costa, Faria \& Reis, 2016).

A compreensão do fenômeno de fixação de ideias na concepção de novos produtos requer o desenvolvimento de instrumentos e procedimentos denominados protocolos que permitam a observação, a análise e a descrição das causas que podem levar os designers a fixar ideias e reproduzir características de produtos existentes no mercado (Camelo et al., 2017). Desta forma, o objetivo do presente trabalho foi apresentar dois protocolos de pesquisa propostos para identificar o grau de influência das informações ambientais sobre o processo criativo de designers, considerando o modelo SAPPHiRE (Sarkar \& Chakrabarti, 2011) como métrica para avaliar os produtos gerados pelos designers.

Os protocolos apresentados neste trabalho foram elaborados buscando definir as ferramentas para duas fases da pesquisa (Figura 1) intitulada "Criatividade e Sustentabilidade" que vem sendo realizada no Laboratório Avançado sobre Estudos em Design e Inovação (LAEDIn) da Universidade Estadual de Maringá (UEM). A primeira fase estabelece o protocolo que permitiu aos pesquisadores aplicar a simulação de prática voltada para o desenvolvimento de produtos, considerando dados ambientais. Os produtos gerados foram registrados de maneira a fornecer dados para a fase seguinte da pesquisa. A segunda fase estabeleceu o procedimento de avaliação a partir do critério 
de criatividade, com objetividade de acordo com a proposta do modelo de avaliação da criatividade SAPPhIRE proposto por Sarkar e Chakrabarti (2011). Este modelo de avaliação foi utilizado por designers especialistas para a obtenção de informações para posterior análise de relação causal entre o nível de detalhamento das informações fornecidas e o grau de criatividade alcançado pelas propostas.

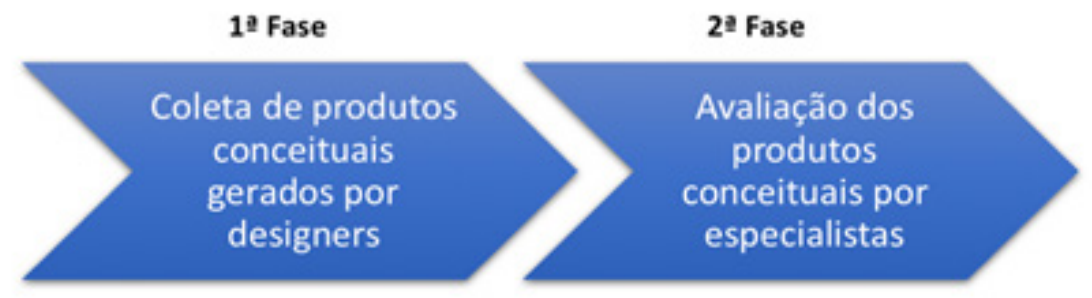

Figura 1 - Etapas estabelecidas para o desenvolvimento do projeto de pesquisa "Criatividade e Sustentabilidade”.

\section{Materiais e métodos}

Para a elaboração dos protocolos de pesquisa foi empregado o método de pesquisa qualitativa, com o intuito de avaliar os instrumentos de coleta de dados sobre o processo de geração de ideias desenvolvidas por equipes compostas por designers, bem como a avaliação das ideias por designers especialistas. Inicialmente foi realizado o projeto piloto, sendo selecionados cinco participantes, quatro designers em processo de formação e um especialista de design, os primeiros utilizaram o protocolo de coleta sobre os produtos conceituais e o especialista empregou o protocolo de avaliação de conceitos gerados. Inicialmente é realizada a descrição do uso dos métodos qualitativos para a elaboração dos protocolos de pesquisa, denominada fase preliminar do projeto, em seguida é descrito o processo de aplicação e análise do projeto piloto de criação de conceitos e, por fim, o processo piloto de avaliação dos conceitos de design.

Esta pesquisa foi aprovada pelo Comitê Permanente de Ética em Pesquisa com Seres Humanos (COPEP) da Universidade Estadual de Maringá no edital 013/2014-COPEP sob o número CAAE 35326514.8.0000.0104.

\subsection{Desenvolvimento dos instrumentos de pesquisa}

A fase preliminar do projeto utilizou o modelo SAPPhIRE proposto por Sakar e Chakrabarti (2011) para avaliar a criatividade de produtos de design. Por meio deste modelo foram definidos critérios mensuráveis para que designers especialistas pudessem avaliar produtos tomando como referência dois eixos: a novidade da proposta e a utilidade deste produto, segundo os ponto de vista da sociedade onde a proposta está inserida. A novidade compreende as características peculiares que surgem quando um produto é comparado com outros existentes no mercado, enquanto a definição de utilidade pode ser entendida como a visão social deste produto e seu uso considerando 
aspectos como a importância e a popularidade do produto, a frequência de uso e o tempo dedicado ao uso do produto (Sakar \& Chakrabarti, 2011).

A atividade posterior foi descrever de forma visual as informações internas e externas dos produtos, como referência para o desenvolvimento simulado de novos produtos e a avaliação comparativa com as propostas criadas pelos designers. Para tanto, foi realizada a desmontagem de dois produtos eletroeletrônicos buscando identificar suas funções internas e externas, os materiais adotados para a fabricação dos componentes e o comportamento entre as peças. Estas informações foram representadas por meio de diagramas e tabelas buscando facilitar a consulta dos designers projetistas e dos avaliadores durante os experimentos de pesquisa. As informações coletadas e complementadas com o trabalho de Ostad-Ahmad-Ghorabi, Huber e Pamminger (2007), foram representadas segundo a proposta do modelo SAPPhIRE e da estrutura de representação Função, Comportamento e Estrutura (Function-Behaviour-Structure FBS) proposto por Gero e Kannengiesser (2004).

Após a análise do modelo teórico de avaliação e dos produtos eletroeletrônicos, as informações, como diagramas de orientação, fluxogramas de atividades e dados funcionais e ambientais dos produtos, foram organizadas para uso nas fases de desenvolvimento dos protocolos.

A primeira fase da pesquisa permitiu definir o protocolo de orientação aos pesquisadores para realizar práticas simuladas de projeto de design fornecendo três níveis de detalhamento de informação ambiental às equipes de designers. Por meio deste protocolo, os resultados das práticas foram registrados e as propostas de projetos conceituais identificadas para sua posterior avaliação pelos designers especialistas. A versão preliminar do protocolo considerou os seguintes instrumentos: (1) Roteiro acompanhado de um checklist das atividades, das leituras e das orientações necessárias para que os pesquisadores pudessem orientar a aplicação da pesquisa aos grupos compostos por designers. Este material foi elaborado como forma de padronizar os procedimentos de aplicação e coleta de dados, evitando as interferências nas práticas de desenvolvimento observadas. (2) Texto informativo contendo dados fictícios sobre a demanda de uma empresa de eletroeletrônicos por projetos de novos produtos. (3) Formulários para que os designers pudessem registrar as ideias elaboradas durante o experimento. Este formulário vem acompanhado de um modelo buscando orientar o preenchimento dos dados sobre o produto que serão necessários para a análise e avaliação dos especialistas. (4) Formulário de autoavaliação para que os designers pudessem identificar em cada proposta de produto, quais foram as melhorias ambientais, quais as contribuições sobre a criatividade de um novo produto, qual seria a viabilidade da proposta e qual o grau de originalidade do produto. (5) E, por fim, uma mini-survey para identificar o perfil dos designers que compõem os grupos de trabalho.

Posteriormente, foi elaborado o protocolo de apoio ao processo de avaliação para ser aplicado nos dois ciclos de desenvolvimento. O primeiro ciclo considerou estabelecer instrumentos para avaliar o critério de novidade dos produtos, enquanto que o segundo ciclo considerou aprimorar esses instrumentos e acrescentar o critério de utilidade do produto. Em ambos ciclos os instrumentos tomaram como referência as informações coletadas na fase preliminar do projeto. As aplicações piloto foram realizadas para cada 
ciclo, as observações de cada sessão e as entrevistas foram gravadas em vídeo. Ao final de cada ciclo, as melhorias foram identificadas e os instrumentos reestruturados para adequá-los a melhor interação com os designers especialistas.

\subsection{Aplicação piloto sobre a criação de novos produtos}

Após a elaboração do roteiro e dos instrumentos de pesquisa para simular o desenvolvimento do projeto foi realizada a aplicação piloto da pesquisa em forma de sessão, gravada em vídeo (Figura 2). Após o registro dos projetos de novos produtos foi aplicada entrevista semiestruturada buscando identificar as dificuldades encontradas pela equipe para interpretar o material fornecido e para entender a lógica do uso dos instrumentos. Os vídeos foram transcritos e analisados pelos pesquisadores, os fragmentos foram separados demonstrando as impressões quanto ao uso do material fornecido durante a pesquisa. A análise dos fragmentos permitiu identificar problemas no protocolo que possibilitou a reformulação para facilitar a interpretação das informações fornecidas e permitir o registro adequado das informações do projeto que seriam analisadas nas fases posteriores da avaliação.

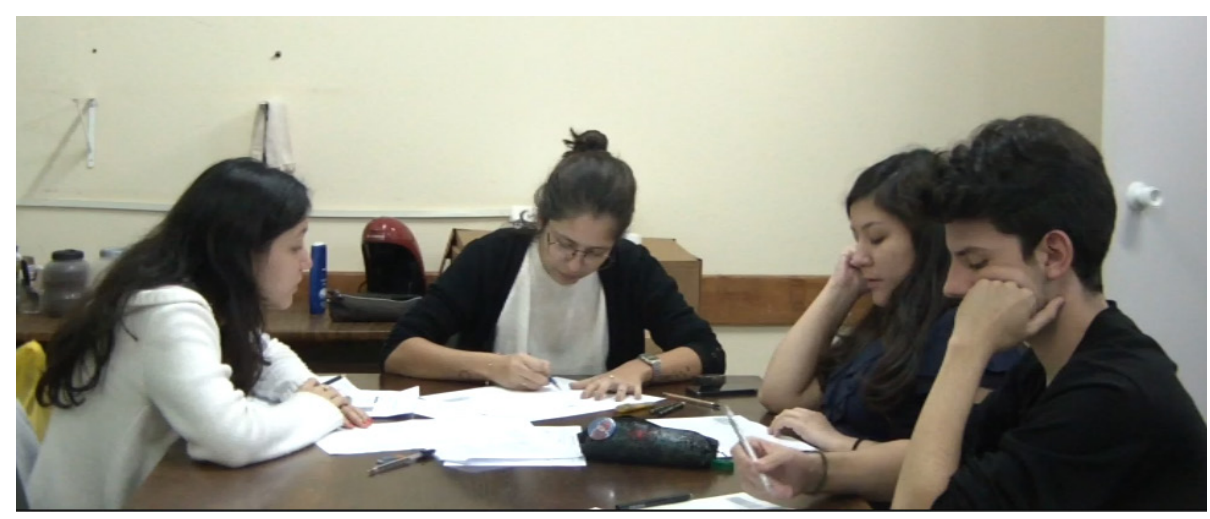

Figura 2 - Grupo de designers desenvolvendo soluções na aplicação piloto de coleta de produtos conceituais.

\subsection{Aplicação piloto do protocolo de avaliação}

No primeiro ciclo de desenvolvimento o protocolo considerou o desenvolvimento dos seguintes instrumentos: roteiro para orientar o pesquisador na aplicação do processo de avaliação, fluxograma apontando os passos necessários para avaliar a novidade do produto analisado, formulários para registrar as pontuações alcançadas por cada proposta analisada e sketches (desenhos) de produtos desenvolvidos para serem analisados pelos especialistas (Figura 3).

No segundo ciclo, o fluxo de avaliação apresentado pelo modelo SAPPhIRE precisou ser readequado para que os cálculos de avaliação pudessem ser realizados pela própria equipe de pesquisa. Com esta modificação, a avaliação pôde ser realizada pelo especialista de forma mais simplificada, adotando somente a atividade de preenchimento dos 


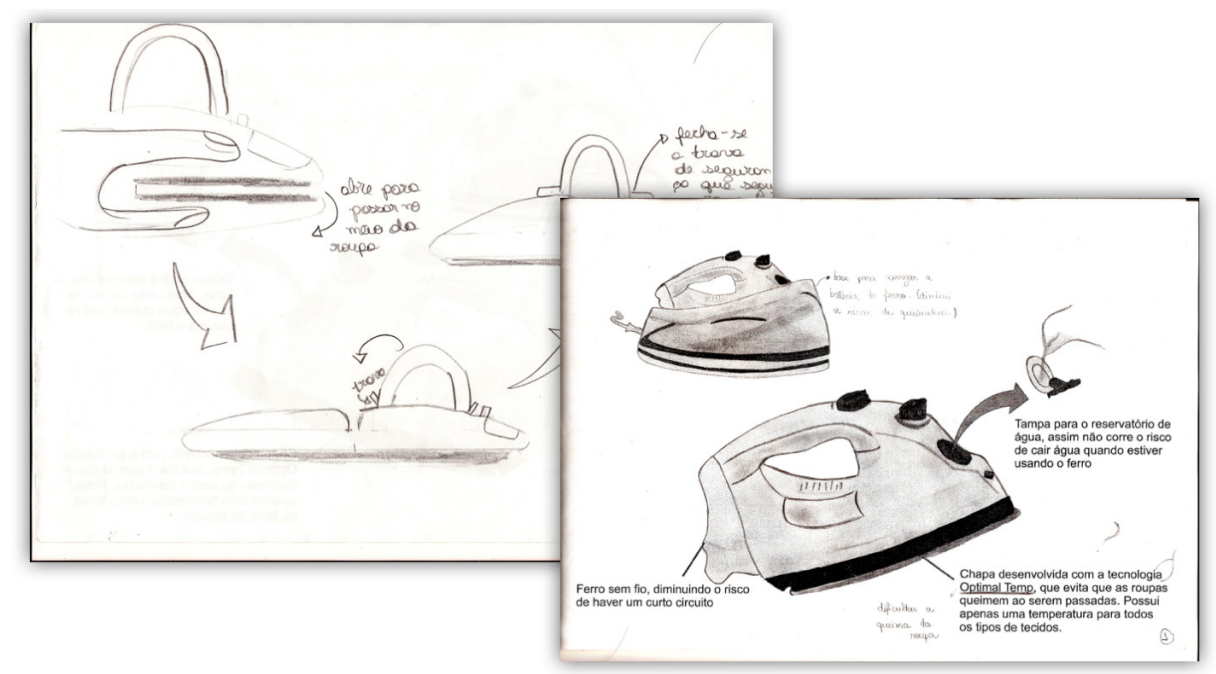

Figura 3 - Desenhos utilizado na pesquisa piloto sobre a avaliação dos produtos conceituais.

formulários para cada produto avaliado. Desta forma, foi possível tornar o processo mais rápido e evitar divergências quanto a pontuação adotada na análise dos produtos. Abaixo, as figuras apresentam a 1a versão dos fluxos de avaliação (Figura 4a) e a $2 a$ versão dos fluxos através de um processo mais simplificado (Figura 4b).

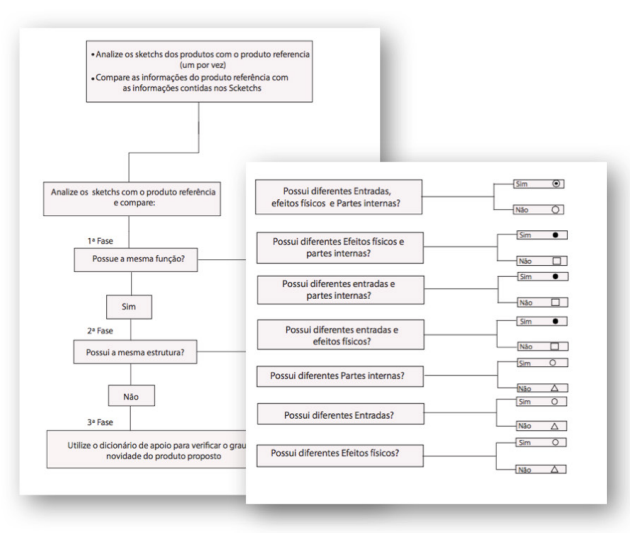

(a)

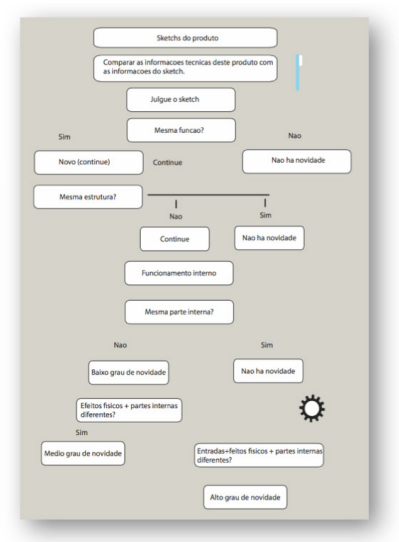

(b)

Figura 4 - Versões dos fluxos de orientação para que o avaliador pudesse analisar e pontuar os produtos desenvolvidos pelos designers.

A aplicação piloto foi conduzida para que o especialista de design pudesse ler e se orientar com os materiais fornecidos e a avaliação pudesse ser conduzida sem a interferência 
dos pesquisadores. Esta prática foi gravada em vídeo e, em seguida, uma entrevista semiestruturada foi realizada para identificar as impressões sobre o material utilizado. Ao longo da entrevista foram questionados sobre quais as dificuldades encontradas para avaliar as propostas, qual informação foi mal interpretada e o que poderia ser melhorados nas ferramentas para que material pudesse ficar mais fácil de entender. $\mathrm{O}$ vídeo desta sessão e da entrevista foram analisados pelos pesquisadores e fragmentos foram separados buscando ilustrar as dificuldades quanto ao uso dos instrumentos, os problemas de interpretação e as sugestões de melhorias. Trechos da entrevista, como o transcrito abaixo, permitiram identificar as dificuldades que o avaliador teve para interpretar partes das instruções. Seus comentários foram combinados com as imagens do vídeo (Figura 5), permitindo mostrar as propostas do próprio avaliador no uso dos formulários.

“(...) Durante a leitura, você tem a sensação de que vai se sair bem, porque o texto parece uma coisa mais simples do que ela se mostrou. Eu achei mais complicado quando eu tive que realmente fazer o que era solicitado.

Quando era explicado parecia algo assim, tudo bem sigo umas etapas e essas etapas vão ser auto explicativas. Mas eu comecei a ter que interpretar, assim. A sensação que as instruções passavam foram diferentes da avaliação a realizar."

Trecho da entrevista realizada com o especialista

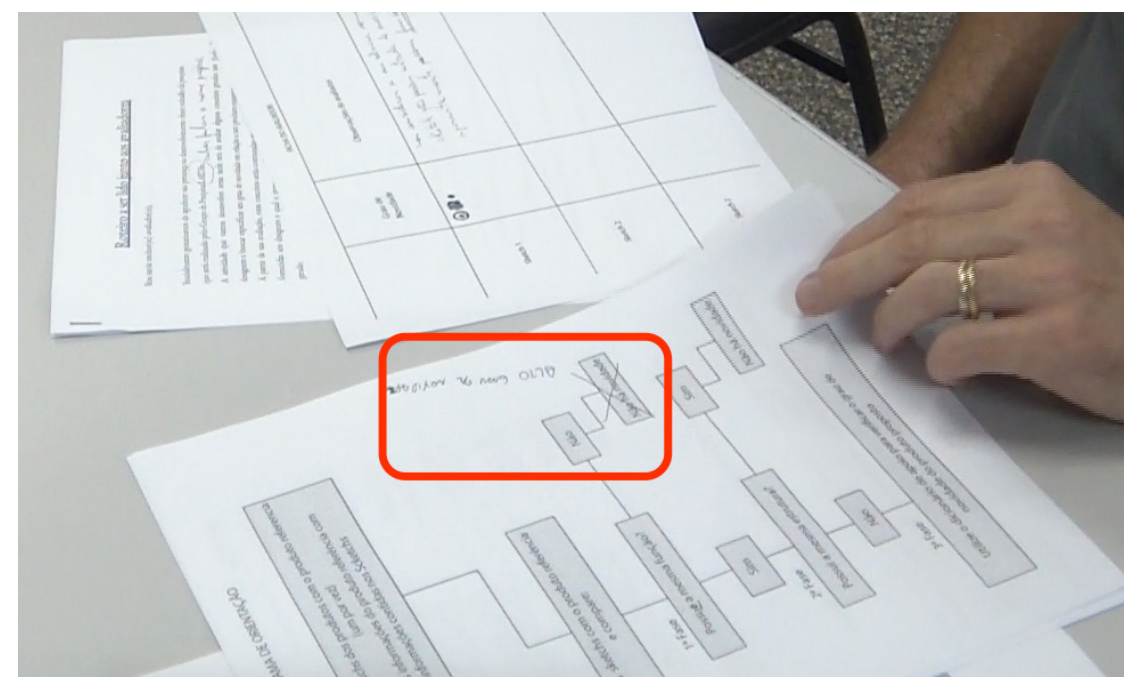

Figura 5 - Trecho da entrevista após a aplicação piloto onde mostra as dificuldades que teve para interpretar o material.

Após a análise dos problemas e comentários feitos ao longo da entrevista, a equipe de pesquisadores aprimorou os instrumentos. Em seguida, uma segunda aplicação piloto foi realizada buscando certificar o material retrabalhado. Nesta nova aplicação 
o avaliador foi observado e novamente entrevistado. Outras necessidades de melhorias foram identificadas para aprimorar o protocolo do projeto, como: facilidade de interpretação das informações, registro adequado das avaliações do projeto e praticidade no uso dos formulários. A Figura 6 apresenta o experimento realizado no 20 ciclo de desenvolvimento.

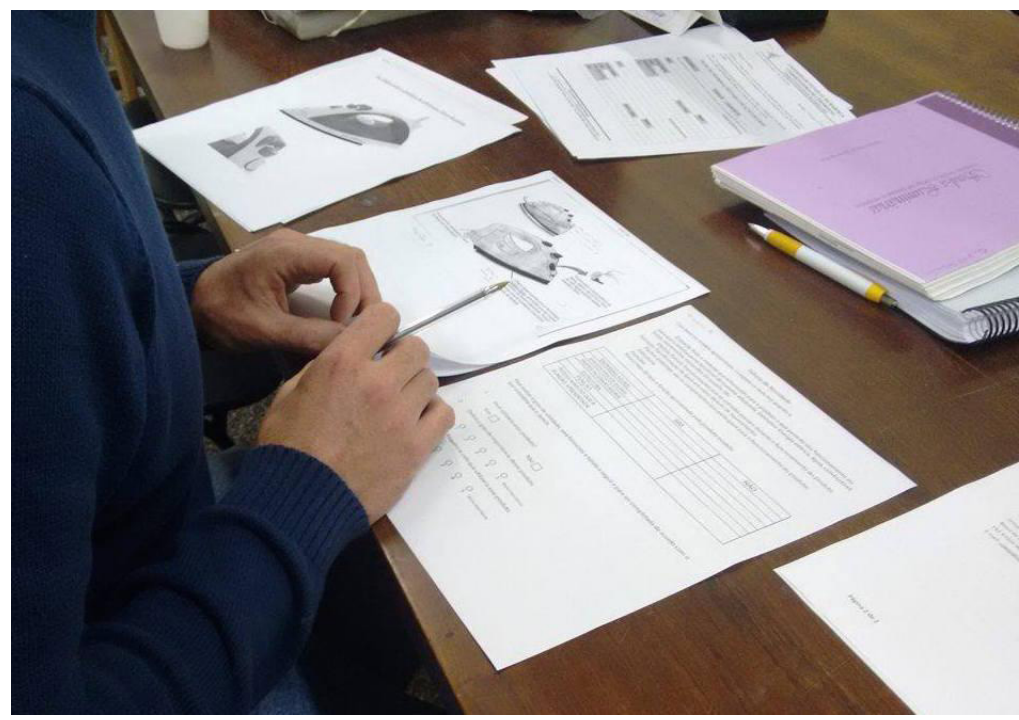

Figura 6 - Aplicação piloto dos instrumentos de suporte a avaliação considerando aspectos da novidade e utilidade dos produtos.

\section{Resultados}

Após as aplicações do projeto piloto, observando, entrevistando e aprofundando o uso dos instrumentos de pesquisa, foram elaborados dois protocolos implementando duas fases do projeto: o protocolo de coleta de produtos conceituais para que designers possam desenvolver projetos de novos produtos em laboratório, e outro protocolo para auxiliar especialistas no processo de avaliação dos conceitos gerados. Para o registro e coleta de dados sobre os produtos desenvolvidos em equipe, os instrumentos elaborados iniciaram com 8 instrumentos com instruções, informações e formulários que foram reduzidos a 6 instrumentos com linguagem mais clara e com poucos diagramas e fluxos, tornando o processo fácil de ser interpretado e seguido (Figura 7a). E, para o registro das avaliações, foram propostos 3 instrumentos (Figura $7 \mathrm{~b}$ ), composto por roteiro de orientação (sem fluxogramas ou diagramas), formulários de avaliação (sem cálculos) onde o especialista marcaria somente os aspectos encontrados e material contendo informações de um produto de referência para que este pudesse ser comparado com as propostas, caso houvesse necessidade. Todo o processo de cálculo das avaliações foi retirado dos formulários de avaliação e passou a ser durante a etapa e análise pelos designers especialistas. Na aplicação definitiva do protocolo de avaliação, espera- 
se considerar a participação de três especialistas em design, cujas avaliações serão calculadas após as análises dos produtos gerados pelos designers.

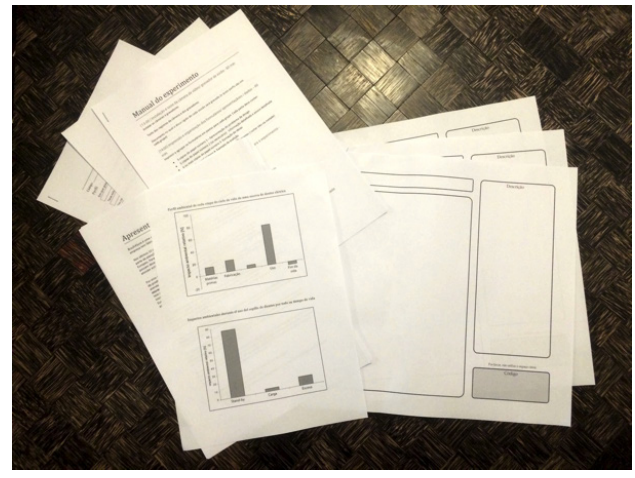

(a)

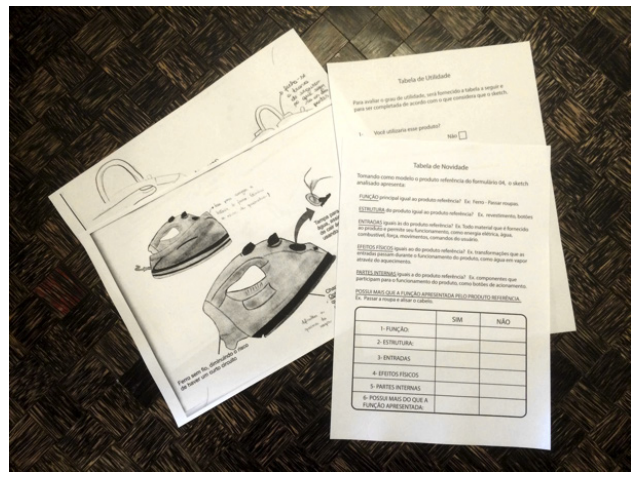

(b)

Figura 7 - Instrumentos aprimorados após análise e identificação das melhorias indicadas pelos participantes da pesquisa.

Quanto ao uso do modelo teórico de avaliação apresentado por Sarkar e Chakrabarti (2011), foram identificadas duas lacunas que foram trabalhadas no projeto de pesquisa. A primeira lacuna dizia respeito a dificuldade de entendimento do modelo de avaliação e a interpretação da nomenclatura por designers. Neste caso, foi necessário transformar os processos gráficos em texto explicativo dos passos necessários para a avaliação. Além disso, foram definidos termos para reduzir a ambiguidade das informações dadas aos designers e ao avaliador. A segunda lacuna dizia respeito ao cálculo adotado para avaliar as propostas dos produtos conceituais. Estes cálculos foram separados dos formulários utilizados pelo avaliador. Assim, os avaliadores poderão ficar focados em responder um formulário para cada produto conceitual e o cálculo seria realizado posteriormente e de maneira automatizada com o auxílio dos pesquisadores.

Ao final, foi possível adequar o modelo estudado às demandas delinguagem, interpretação e praticidades observadas através das dificuldades dos designers e do especialista em utilizados os instrumentos da pesquisa. Desta forma foi possível elaborar protocolos mais simples e claros.

\section{Conclusões}

O trabalho desenvolvido pelo grupo de pesquisa LAEDIn permitiu identificar a importância da pesquisa qualitativa no desenvolvimento de instrumentos de pesquisas para o design. Por meio de ciclos de experimentação, observação, entrevistas e análises foi possível identificar problemas, limitações e oportunidades que permitiram aprimorar os protocolos para a pesquisa "Criatividade e Sustentabilidade". Para definir estes protocolos, o presente estudo apresentou a criação de instrumentos adaptados do modelo de avaliação da criatividade SAPPhIRE (Sarkar \& Chakrabarti, 2011). 
Sobre a elaboração dos protocolos, o uso do método qualitativo permitiu analisar e identificar falhas e dificuldades no uso de instrumentos de pesquisa a partir de projetos pilotos gravados em vídeo e aplicação de entrevistas semiestruturadas com os integrantes das equipes. As falhas foram corrigidas e novos instrumentos foram propostos buscando aumentar a facilidade do uso, permitindo interação entre os participantes do projeto e simplificando o processo de análise do material coletado.

Espera-se que em breve estes protocolos desenvolvidos possam ser aplicados em sessões definitivas de criação envolvendo outras equipes de designers e nas sessões de avaliação das propostas contando com a colaboração de especialistas de design. Após realizada a coleta de dados, espera-se dar seguimento às fases seguintes do projeto de pesquisa buscando identificar a relação de causa e efeito entre a informação ambiental de produtos de design em diferentes níveis de detalhamento com o grau de criatividade a ser alcançado pelas propostas de produtos criadas por equipes de designers.

\section{Referências}

Camelo, D. M., Marques, A. G., Roque, A., Vieira, A., \& Bellido, I. (2017) O uso de métodos qualitativos para o desenvolvimento de instrumentos de avaliação da criatividade em produtos conceituais de design. Atas do $6^{\circ}$ CIAIQ 2017 - Investigação Qualitativa em Engenharia e Tecnologia. V. 4. Salamanca, Espanha.

Collado-Ruiz, D., \& Ostad-Ahmad-Ghorabi, H. (2010). Influence of Environmental Information on Creativity. Design Studies, 31(5), 479-498. doi: 10.1016/j. destud.2010.06.005

Contreras, J., Moya, J., Melo, M., \& Arias, J. (2017) Aplicación de metodologías mistas para la mejora de materiales educativos universitarios. Atas do 6 o CIAIQ 2017 Investigação Qualitativa em Engenharia e Tecnologia. V. 4. Salamanca, Espanha.

Costa, A. P., Faria, B. M., \& Reis, L. P. (2016) Investigação Qualitativa Através da Utilização de Software: Workflows Metodoógicos. RISTI - Revista Ibérica de Sistemas e Tecnologías de Informação, (19), ix-xii.

Crilly, N. (2015). Fixation and Creativity in Concept Development: The Attitudes and Practices of Expert Designers. Design Studies, 38, 54-91. doi: 10.1016/j. destud.2015.01.002

Daros, C., Camelo, D., Silvério, \& D., Santos A. (2012) Avaliação qualitativa de hábitos de consumo: resultados preliminares a partir do método das Sondas Culturais. Anais do 40 Encontro de Coordenadores da Rede de Pesquisa sobre o uso racional de água e eficiência energética em Habitações de Interesse Social. p. 35-76. Editora UFS. São Cristóvão, Sergipe, Brasil.

García-Merino, T., (2017) Vídeos en la investigación en dirección de empresas: Su estudio mediante análisis del discurso y de la cognición corporal. Atas do 6o CIAIQ 2017 - Investigação Qualitativa em Ciências Sociais. V.3. Salamanca, Espanha.

Gero, J. S., \& Kannengiesser, U. (2004). The Situated Function-Behaviour-Structure Framework. Design Studies, 25(4), 373-391. doi: 10.1016/j.destud.2003.10.010 
Lopez-Mesa, B., Mulet, E., Vidal, R., \& Thompson, G. (2011). Effects of Additional Stimuli on Idea-Finding in Design Teams. Journal of Engineering Design, 22(1), 31-54. doi: 10.1080/09544820902911366

Ostad-Ahmad-Ghorabi, H., Huber, M., \& Pamminger, R. (2007) Ecodesign for Sustainable Development. Product Development. In: W. Wimmer (editor). V.4. Brasov: Transilvania University of Brasov.

Purcell, A. T., \& Gero, J. S. (1996). Design and Other Types of Fixation. Design studies, 17(4), 363-383. doi: 10.1016/So142-694X(96)ooo23-3

Sarkar, P., \& Chakrabarti, A. (2011). Assessing design creativity. Design Studies, 32(4), 348-383. doi: 10.1016/j.destud.2011.01.002

Silva, G., \& Correa, M. (2017). Videoanálise na pesquisa e na formação inicial de professores de Física. Atas do 60 CIAIQ 2017 - Investigação Qualitativa em Educação. V. 1. Salamanca, Espanha.

Vosgerau, D. S. R., Pocrifka, D. H., \& Simonian, M., (2016). Associação entre a técnica de análise de conteúdo e os ciclos de codificação: possibilidades a partir do software ATLAS.ti. RISTI - Revista Ibérica de Sistemas e Tecnologías de Informação, (19), 93-106. 